\title{
Effect of Skin Tumor Properties on Laser Penetration
}

\author{
A E Karsten*a,b, A Singh ${ }^{\mathrm{a}}$ \\ ${ }^{a}$ Biophotonics Group, National Laser Centre, Council for Scientific and Industrial Research, \\ P. O. Box 395, Pretoria, 0001, South Africa. \\ ${ }^{\mathrm{b}}$ Physics Department, Faculty of Natural and Agricultural Sciences, University of Pretoria, Pretoria, \\ 0002, South Africa.
}

\begin{abstract}
Computer modeling can be a valuable tool to determine the absorption of laser light in different skin layers. For this study, the optical properties of three different skin tumors were used in the model to evaluate the effect on penetration depth into the skin. Comparison between the healthy dermis and the skin tumors indicated that up to $28 \%$ more laser light is absorbed in the healthy dermis than in the tumor tissue. This has implications on the laser dose applied to the skin for treatment.
\end{abstract}

Keywords: Light interaction with tissue, laser, laser treatment, dosimetry, modeling.

\section{INTRODUCTION}

Worldwide the trend in medicine is to move to minimal (or less) invasive treatments and diagnostic modalities. Using lasers in the treatment of diseases such as cancer, has definite advantages. It is however essential to understand the limitations of laser as a treatment modality. Before any treatment is attempted, it is important to know the optical properties of all the tissues types/layers that will be irradiated by the laser as well as the properties of the laser source itself. With the information available it is possible to predict the absorption of the laser light at different depths through the skin (or other organ). These results can help the medical practitioner to treat a patient with minimal damage to the surrounding healthy tissue.

Photodynamic therapy (PDT) is one of the current laser technologies available for treating cancer. This therapy is a treatment modality where a photosensitiser (PS) is administered to a patient and after a period of time the tumor is irradiated with a laser, tuned to an absorption wavelength of the PS. In order to deliver the correct amount of laser power to the tumor, it is important to understand the absorption and scattering of laser light in the tissue. The absorption and scattering properties is a function of the tissue type and the laser wavelength. If the tumor is sub-surface, the penetration through the outer skin layers must be taken into account when the treatment parameters are specified. The optical properties for different skin types are not the same and therefore influence the amount of laser light reaching the treatment area. The radiant exposure $(\mathrm{H})$ is used in PDT to specify the surface irradiation. Although the SI unit is $\mathrm{J} / \mathrm{m}^{2}$, it is more common to use $\mathrm{J} / \mathrm{cm}^{2}$ as the unit ${ }^{[1]}$. The "dose" (specified in $\mathrm{J} / \mathrm{cm}^{2}$ ) is normally specified for treatment.

In developing PDT as a treatment modality, modeling of the interaction of the laser light with the tissue is a valuable tool to predict the radiant exposure at the treatment site. It also allows the researcher to investigate the effect of variations in the laser parameters (e.g. change in laser power of beam diameter) in a fast and cost effective manner without the need for skin samples/patients. Extreme parameters can also be tested without harm to patients.

\subsection{Light penetration modeling}

In order to model the propagation of the light into the skin, the characteristics of the laser source and the optical properties of the tissue or skin must be known. Due to the selectivity of the PDT process, a laser with a specific wavelength is selected (normally between $620 \mathrm{~nm}-685 \mathrm{~nm}$ ), depending on the drug or PS being used. The optical properties: absorption coefficient $\left(\mu_{\mathrm{a}}\right)$, scattering coefficient $\left(\mu_{\mathrm{s}}\right)$, anisotropy factor $(\mathrm{g})$ and the refractive index $(\mathrm{n})$ of the skin at the treatment wavelength must be measured or if that is not possible, data from literature can be used. One of the

*AKarsten@esir.co.za; phone +(27)12 841 3428; fax +(27)12 8413152

Therapeutic Laser Applications and Laser-Tissue Interactions IV, edited by Ronald Sroka, Lothar D. Lilge,

Proc. of SPIE-OSA Biomedical Optics, SPIE Vol. 7373, 737316 - ( ) 2009 SPIE-OSA

CCC code: $1605-7422 / 09 / \$ 18 \cdot$ doi: $10.1117 / 12.831948$ 
measurement techniques available for determining the optical properties of a tissue sample is an Integrating Sphere $(\mathrm{IS})^{[2]}$, which can be used to determine the absorption coefficient and the reduced scattering coefficient $\left(\mu_{\mathrm{s}}^{\prime}\right)$. The relationship between $\mu_{\mathrm{s}}^{\prime}$ and $\mu_{\mathrm{s}}$ is given in Eq.1.

$$
\mu_{\mathrm{s}}^{\prime}=(1-\mathrm{g}) \mu_{\mathrm{s}}
$$

The IS setup offers an advantage to most other systems in that it can be used to extract all the optical properties $\left(\mu_{\mathrm{s}}, \mu_{\mathrm{s}}^{\prime}\right.$, $\mu_{\mathrm{a}}$ and $\mathrm{g}$ ) except for $\mathrm{n}$.

For this work $\mathrm{ASAP}^{[3]}$ software was used to model the penetration of light through a slab tissue model, using the optical properties available. The software package is a non-sequential raytracing package that is suited for complex optical systems where scattering and stray light characteristics must be known and controlled ${ }^{[4]}$.

\section{METHODOLOGY}

A layered tissue model was created consisting of a thin epidermal layer, a dermal layer and a "tumor" that was imbedded in the dermal layer. The tumor was imbedded $0.5 \mathrm{~mm}$ from the top or epidermal layer. The total dimensions of the model were $20 \mathrm{~mm}$ x $20 \mathrm{~mm} \times 4.15 \mathrm{~mm}(\mathrm{X}, \mathrm{Y}, \mathrm{Z})$. A graphical presentation of the model is given in Figure 1. Two different laser wavelengths, $630 \mathrm{~nm}$ and $680 \mathrm{~nm}$ (both within the absorption features of a typical PS) were used in the evaluations. The optical properties at the two wavelengths as well as the tissue parameters are listed in Table 1. For completeness, the total attenuation coefficient $\left(\mu_{\mathrm{t}}=\mu_{\mathrm{a}}+\mu_{\mathrm{s}}\right)$ is also listed in Table 1. The model assumes that the optical properties are uniform within each layer. The thickness of each layer is given by $\mathrm{d}(\mathrm{mm})$. A $1 \mathrm{~mW}$ fibre laser with a beam diameter of $10 \mathrm{~mm}$ was used as the illumination source for the tissue. In the simulations 786997 photons were traced through the model using the Monte Carlo method to determine the trajectory and/or absorption of each photon through the model. The trajectory of a photon is traced until it is absorbed in the model or leaves the boundaries of the model.

The optical parameters used in the model were taken from ${ }^{[5],[6]}$. Three different non-melanoma skin cancers: nodular basal cell carcinoma (NBCC), infiltrative basal cell carcinoma (IBCC) and squamous cell carcinoma (SCC) were investigated. In the "control" model, the properties of the tumor were kept the same as for the surrounding dermal layer. The absorption of light in the epidermal layer is a function of the color of the skin or skin tone. This effect is very important in the dose calculations and has been described previously ${ }^{[7]}$ and not taken into account for these evaluations. The aim of this study is to investigate the absorption of light in the different tumors before the PS is added to the tumor. The addition of the PS into the tissue will change the optical properties.

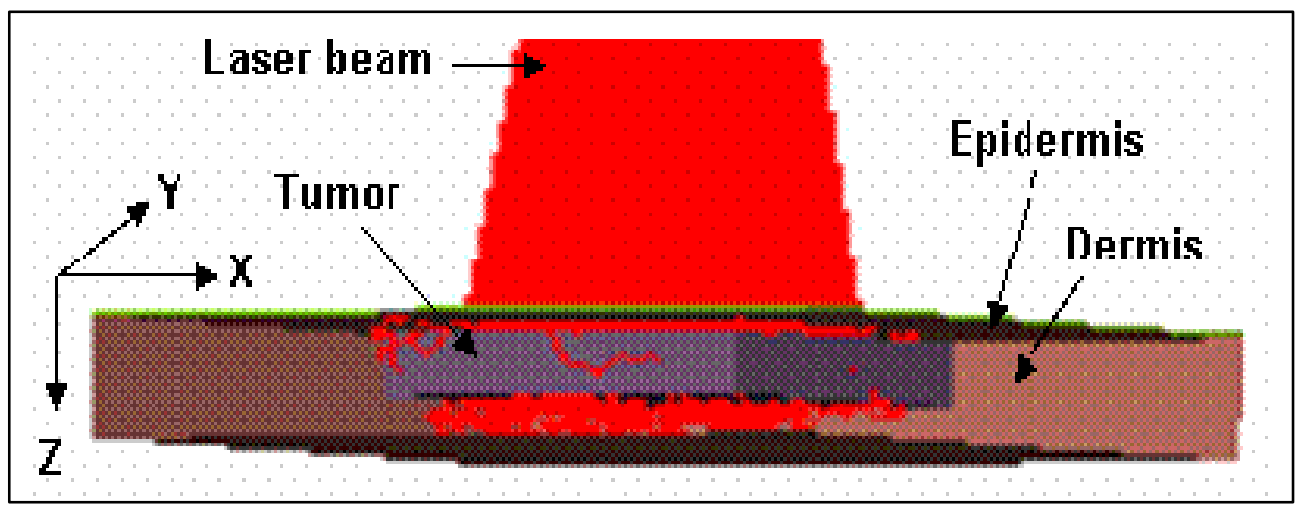

Figure 1: Model of the skin with the tumor imbedded in the dermis. 
Table 1: Optical properties used in the model.

\begin{tabular}{|c|c|c|c|c|c|c|c|c|c|}
\hline \multirow[b]{2}{*}{ Name } & \multirow[b]{2}{*}{ g } & \multirow[b]{2}{*}{$\mathbf{n}$} & \multirow{2}{*}{$\underset{(\mathbf{m m})}{\mathbf{d}}$} & \multicolumn{3}{|c|}{$\lambda=630 \mathrm{~nm}$} & \multicolumn{3}{|c|}{$\lambda=680 \mathrm{~nm}$} \\
\hline & & & & $\begin{array}{c}\mu_{\mathrm{s}} \\
\left(\mathbf{m m}^{-1}\right)\end{array}$ & $\begin{array}{c}\mu_{\mathrm{a}} \\
\left(\mathbf{m m}^{-1}\right)\end{array}$ & $\begin{array}{c}\boldsymbol{\mu}_{\mathrm{t}} \\
\left(\mathbf{m m}^{-1}\right)\end{array}$ & $\begin{array}{c}\boldsymbol{\mu}_{\mathrm{s}} \\
\left(\mathbf{m m}^{-1}\right)\end{array}$ & $\begin{array}{c}\mu_{\mathrm{a}} \\
\left(\mathrm{mm}^{-1}\right)\end{array}$ & $\begin{array}{c}\mu_{\mathrm{t}} \\
\left(\mathbf{m m}^{-1}\right)\end{array}$ \\
\hline Epidermis & 0.79 & 1.5 & 0.15 & 22.86 & 0.27 & 23.13 & 20.95 & 0.27 & 21.22 \\
\hline Dermis & 0.82 & 1.4 & 4 & 16.94 & 0.16 & 17.10 & 13.33 & 0.16 & 13.49 \\
\hline IBCC & 0.80 & 1.4 & 2 & 14.25 & 0.16 & 14.41 & 13.0 & 0.15 & 13.15 \\
\hline NBCC & 0.80 & 1.4 & 2 & 11.5 & 0.13 & 11.63 & 10.25 & 0.09 & 10.34 \\
\hline SCC & 0.80 & 1.4 & 2 & 9.5 & 0.11 & 9.61 & 8.75 & 0.14 & 8.89 \\
\hline
\end{tabular}

During the evaluations the photons entering the model were traced and presented in "layers" or slices. The thickness (Zaxis) of the model was divided in 150 layers and each layer consists of a set of 150x150 voxels (three dimensional pixels). The number of photons absorbed in each voxel is counted to determine the absorption in each layer in the model.

To illustrate the difference between healthy and tumerous tissue, bulk breast skin samples of 59 year old female Caucasian patient were measured with the IS setup that was described previously ${ }^{[2]}$. Samples were frozen within 6 hours of removal and allowed to thaw out overnight before the measurements were done. Measurements were done from the epidermal side as well as from the fatty side. The results are given in Table 2 and showed positive agreement with some of the published data ${ }^{[5]}$. The measured reduced scattering coefficient was within $13 \%$ to the published data, whereas the absorption coefficient was significantly higher. This may be due to the state of the sample when measured. These results however indicate the need to measure these optical properties in situ. The measurements were done on bulk samples but in future samples will be separated into the different skin layers for more accurate optical property measurements.

Table 2: Measured optical properties of skin at $633 \mathrm{~nm}(*), 630 \mathrm{~nm}\left({ }^{\#}\right)$ and $625 \mathrm{~nm}\left({ }^{\$}\right)$.

\begin{tabular}{|l|l|l|}
\hline Sample & $\boldsymbol{\mu}_{\mathbf{a}}\left(\mathbf{m m}^{-1}\right)$ & $\left.\boldsymbol{\mu}_{\mathbf{s}} \mathbf{~}^{-1} \mathbf{m m}^{-\mathbf{1}}\right)$ \\
\hline Breast skin (epidermal side)* & 0.015 & 1.9 \\
\hline Breast skin (fatty side)* & 0.031 & 1.62 \\
\hline Breast $\operatorname{skin}^{[5], \$}$ & 0.006 & 1.43 \\
\hline Breast cancer ${ }^{[5], \#}$ & 0.31 & 9.41 \\
\hline
\end{tabular}

\section{RESULTS AND DISCUSSIONS}

When the laser beam propagates through the model, the intensity profile of the laser beam changes as indicated in Figures 2 and 3. To illustrate the effect, data from the NBCC tumor (Figure 2) and the 'no tumor' (Figure 3) results at a wavelength of $680 \mathrm{~nm}$ were used. This effect is the result of the scattering and absorption of the laser beam. In Figures 2(a),(b) and 3(a),(b) there is very little spreading of the beam. As the beam progress through the layers, it spreads more due to the scattering properties of the tissue. When evaluating the spreading of the beam, it should be remembered that the distance between the first and last image is only about $2.1 \mathrm{~mm}$. In the first $3 \mathrm{~mm}$ into the skin, the beam profile changes from a "top-hat" profile (equal intensity through the width of the beam) to a Gaussian type profile with substantial variation in the laser intensity through the width of the beam. 


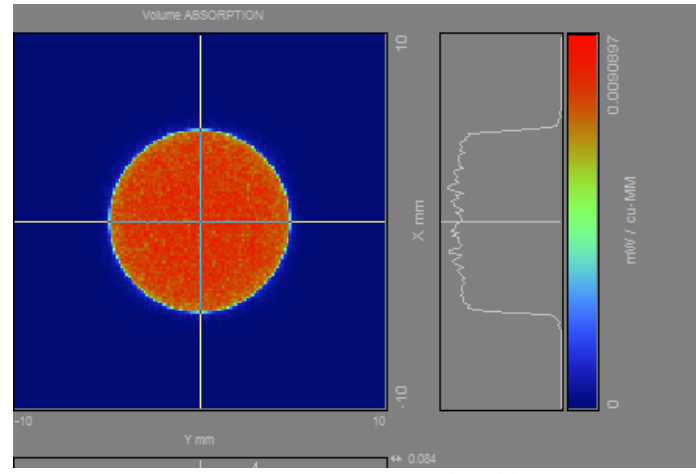

a) In epidermis $(\mathrm{z}=\mathbf{0 . 0 1 3 3} \mathrm{mm})$.

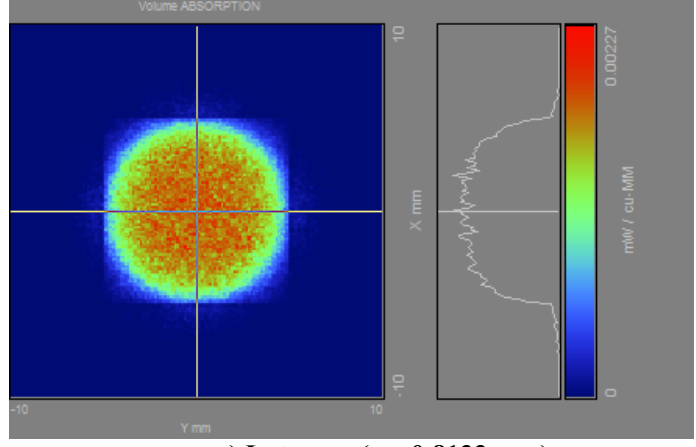

c) In tumor $(\mathrm{z}=\mathbf{0 . 8 1 3 3} \mathrm{mm})$.

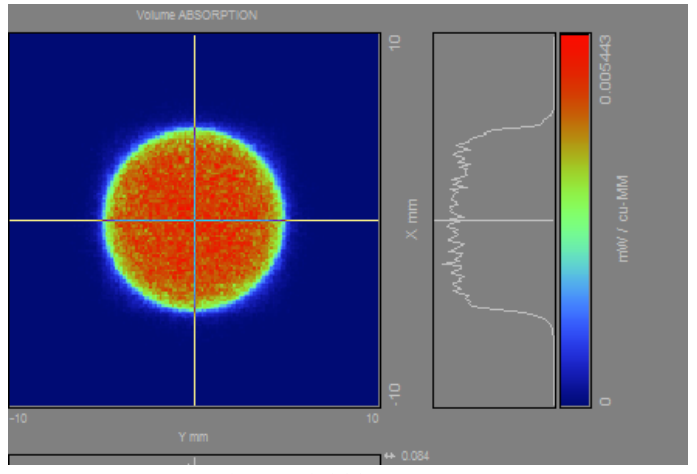

b) In dermis $(\mathrm{z}=0.280 \mathrm{~mm})$.

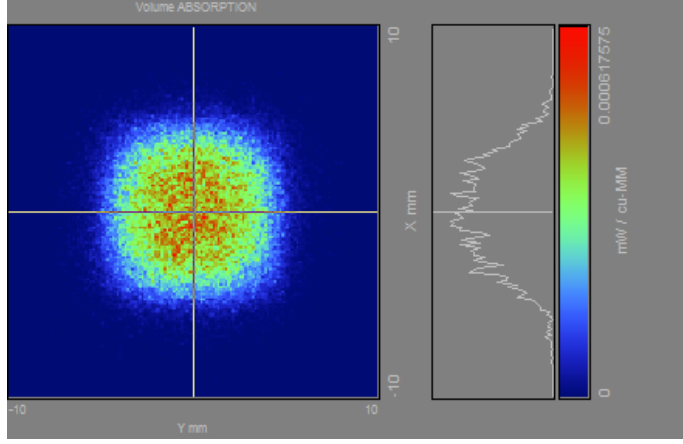

d) In dermis $(\mathrm{z}=\mathbf{2 . 9 4 6 7} \mathrm{mm})$.

Figure 2: NBCC. XY slices of the absorbed light in the model. Slices are shown for different depths starting from the epidermis, dermis, through the tumor and into the bottom part of the dermis after the tumor.

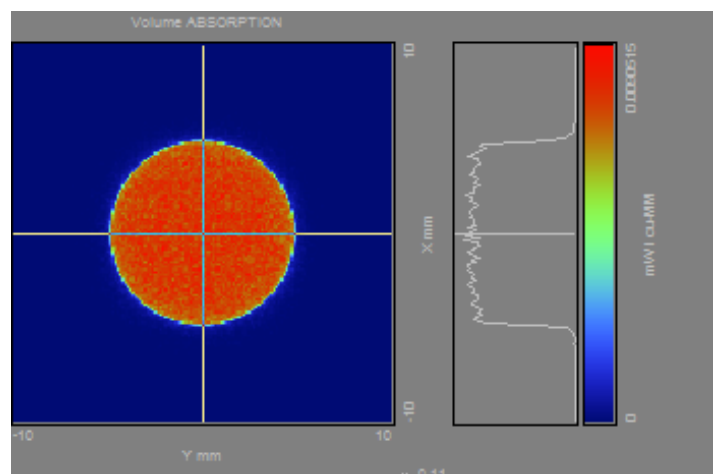

a) In epidermis $(\mathrm{z}=\mathbf{0 . 0 1 3 3} \mathrm{mm})$.

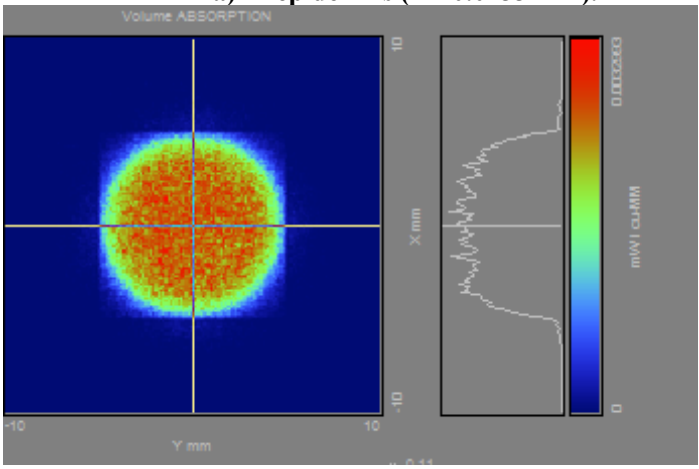

c) In tumor $(\mathrm{z}=\mathbf{0 . 8 1 3 3} \mathrm{mm})$.

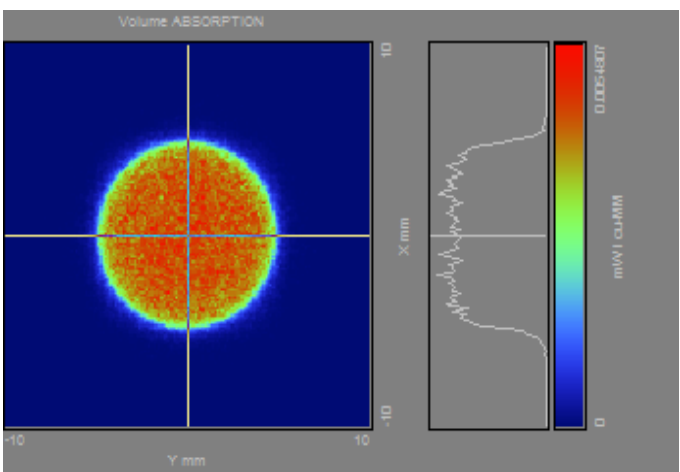

b) In dermis $(\mathrm{z}=\mathbf{0 . 2 8 0} \mathrm{mm})$.

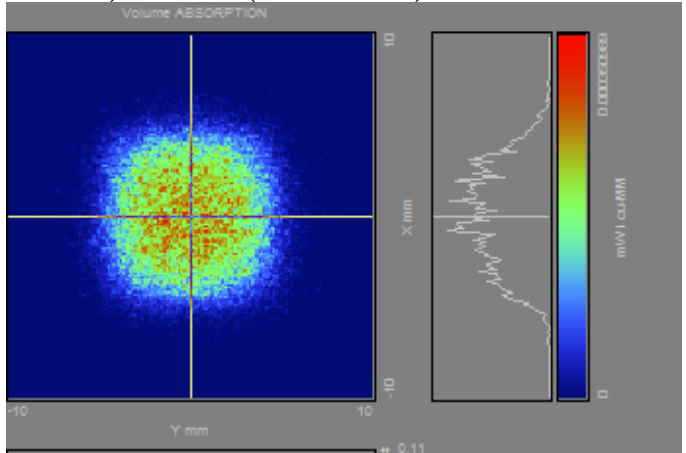

d) In dermis $(\mathrm{z}=\mathbf{2 . 9 4 6 7} \mathrm{mm})$

Figure 3: No tumor. XY slices of the absorbed light in the model. Slices are shown for different depths starting from the epidermis, dermis, the region were the tumor would be and into the bottom part of the dermis. 
In Figures 4 and 5 the absorption values for a few selected layers (different depths) through the model is shown for the two different wavelengths. As the laser beam enters the tumor, the absorption values start to change between the different tumors. The accumulated absorption in the tumors is calculated and presented in Table 3. For both laser wavelengths, the absorption in the dermis (no tumor) and IBCC tumor is very similar. This is evident from Figures 4 and 5 as well as from the table of accumulated absorption (Table 3). The other two tumors (NBCC and SCC) have less absorption than the dermis. At the $630 \mathrm{~nm}$ wavelength the absorption through the SCC tumor is at least $18 \%$ less than that of the dermis and at $680 \mathrm{~nm}$ the NBCC tumor absorbs $28 \%$ less than the dermis. This implies that more laser power is required to provide effective treatment of these tumors if they are imbedded $0.5 \mathrm{~mm}$ under the surface of the skin.

Table 3: Comparison of the accumulated absorbed light in the different tumors (mW).

\begin{tabular}{|c|c|c|c|c|c|c|c|}
\hline $\begin{array}{c}\text { Wavelength } \\
\text { (nm) }\end{array}$ & $\begin{array}{c}\text { No } \\
\text { Tumor }\end{array}$ & IBCC & $\begin{array}{c}\text { \% } \\
\text { Change }\end{array}$ & NBCC & $\begin{array}{c}\text { \% } \\
\text { Change }\end{array}$ & SCC & $\begin{array}{c}\text { \% } \\
\text { Change }\end{array}$ \\
\hline 630 & 0.190 & 0.191 & 5.2 & 0.175 & 7.9 & 0.155 & 18.4 \\
\hline 680 & 0.210 & 0.201 & 4.2 & 0.151 & 28.1 & 0.195 & 7.1 \\
\hline
\end{tabular}

The data presented here did not take the effect of the PS into account. The next step will be to add the PS to the tissue samples. The optical properties of the optimal PS concentration, the sample and the combination of the PS injected in the sample will be determined with an IS system. These values will add accuracy to the model and improve the predictions on the absorption profile as a function of depth into the tissue sample.

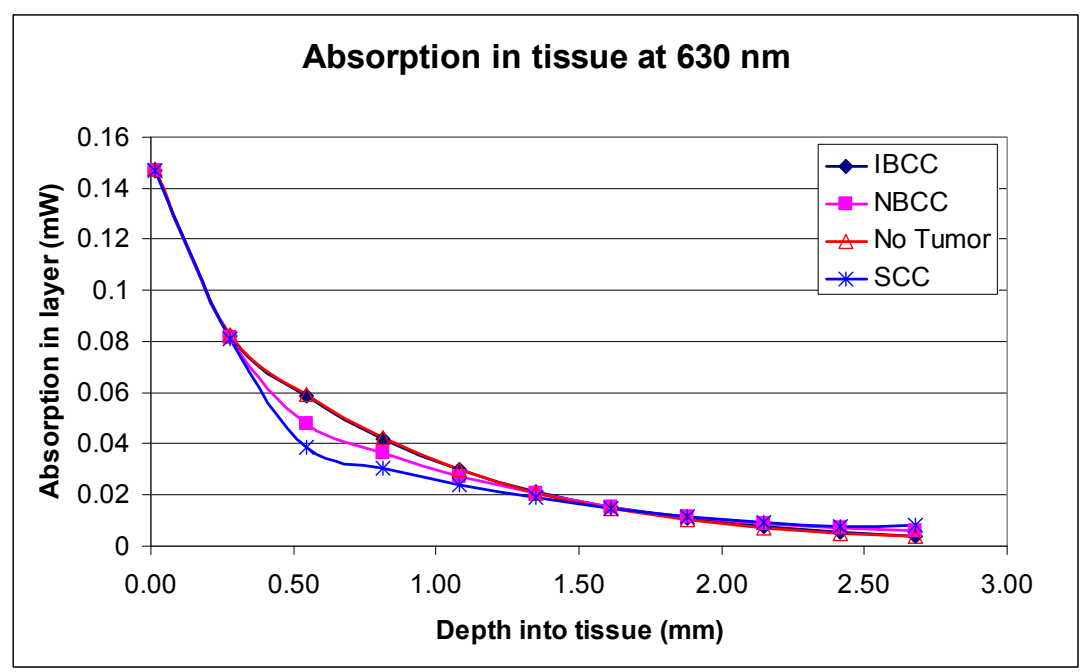

Figure 4: Absorption in the different tumors as a function of depth into the tissue for $\lambda=630 \mathrm{~nm}$. 


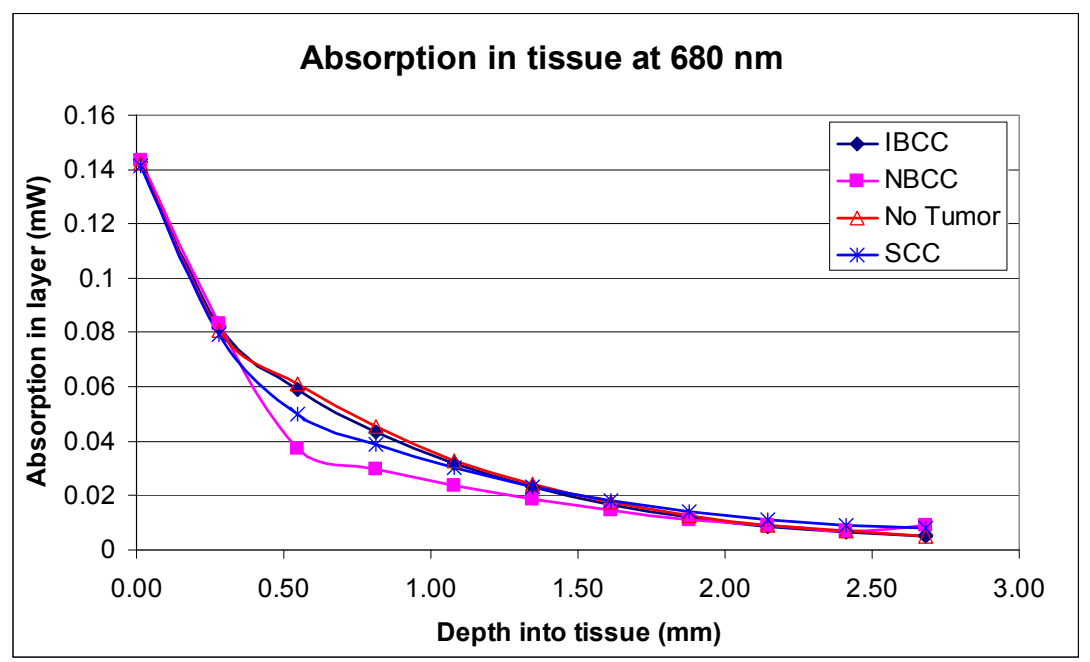

Figure 5: Absorption in the different tumors as a function of depth into the tissue for $\lambda=680 \mathrm{~nm}$.

\section{CONCLUSIONS}

The data presented clearly show that the different tumor properties affect the amount of laser light that is available for absorption in the different layers in the tissue. For the same input laser power, up to $28 \%$ less laser power is absorbed in the tumor when compared to normal tissue. In order to deposit the same amount of photons, more laser light should be applied on the skin to allow for enough photons to reach the tumor. This underlines the fact that the optical properties of the tissue that will be treated must be known beforehand. The software can be used to determine the optimal laser parameters for the specific tumor and the depth of treatment. When determining the treatment parameters, the safety aspects should be taken into account so as not to cause harm to healthy tissue surrounding the tumor.

Numerous authors have published data on optical properties ${ }^{[5]}$, but the data is not always consistent. Variations between the different sets of published data may be due to the variation in time lapse between the time the sample was taken and the measurement time as well as other "stressors" that the tissue may be exposed to. In skin samples the skin tone also has an effect on the measurements. For the most accurate results, a sample similar (skin colour and thickness) to that of the treatment area must be measured to ensure accurate data for modeling. Such work is planned for the near future.

\section{REFERENCES}

[1] "Photodynamic Therapy Dosimetry," AAPM Report no 88, Published for the American Association of Physicists in Medicine by Medical Physics publishing, ISBN-13: 978-1-888340-51-8 (2005).

[2] Singh, A., Karsten, A. E. and Dam, J.S., "Robustness and Accuracy of the Calibration Model for the Determination of the Optical Properties of Chicken Skin," International Conference of the World Association of Laser Therapy, South Africa, 165-169 (2008).

[3] ASAP Software from Breault Research Organization, Inc, Tucson, Arizona.

[4] Stevenson, M., "Optical software: which program is right for me?", OLE, 29-3 (Jul/Aug 2006).

[5] Tuchin,V., [Tissue Optics], SPIE Press (2000).

[6] Salomatina, E., Jiang, B., Novak, J., Yaroslavsky, A.N., "Optical properties of normal and cancerous human skin in the visible and near-infrared spectral range," J. of Biomedical Opt. 11(6) 064026 (2006).

[7] Karsten, A. E., "Effect of Wavelength, Epidermal Thickness and Skin Type on the Required Dose for Photodynamic Therapy," International Conference of the World Association of Laser Therapy, South Africa, 137-143 (2008). 